\title{
Galactic orbits of Planetary Nebulae unveil thin and thick disk populations and cast light on interaction with the interstellar medium
}

\author{
F. Kerber ${ }^{1}$, R. P. Mignani ${ }^{2}$, E.-M. Pauli ${ }^{3}$, A. Wicenec ${ }^{2}$, and F. Guglielmetti ${ }^{4}$ \\ 1 Space Telescope - European Coordinating Facility, Karl-Schwarzschild-Straße 2, 85748 Garching, Germany \\ 2 European Southern Observatory, Karl-Schwarzschild-Str. 2, 85748, Garching, Germany \\ 3 Dr.-Remeis-Sternwarte, Sternwartstraße 7, 96049 Bamberg, Germany \\ 4 Max-Planck-Institut für Plasmaphysik, Boltzmannstrasse 2, 85748, Garching, Germany
}

Received 6 February 2004 / Accepted 30 March 2004

\begin{abstract}
We report reliable proper motion values for the central stars of four Planetary Nebulae (PNe). The proper motions have been compiled from existing optical catalogues i.e. Tycho-2, UCAC2, USNO-B and GSC-II, which are the product of large scale surveys. Results from the different sources have been compared and excellent agreement has been found in all cases reported here. Using known PNe distances and radial velocities, we then computed their Galactic velocity components which, by using a simplified model of the Galactic gravitational field, have allowed us to derive, for the first time, their Galactic orbits. These have provided the first kinematic evidence of the existence of thin and thick Galactic disk PNe populations. This approach, extended to a larger sample of PNe, will result in a statistically more solid basis. For our four individual objects though, the determination of the spatial velocity vector has already provided useful insights into their interaction with the ambient interstellar medium (ISM), confirming that the motion of the central star and the nebular shell through the ISM is the root cause for the interaction process. Our results therefore show how a more quantitative understanding of the interaction process can be achieved in the future. This would provide new insight into the return of metal enriched matter to the ISM, which in turn is in no small part responsible for the chemical evolution of galaxies.
\end{abstract}

Key words. Galaxy: kinematics and dynamics - ISM: evolution - ISM: planetary nebulae: individual: NGC 7293 ISM: planetary nebulae: individual: Sh 2-216 - ISM: planetary nebulae: individual: IC 4593 -

ISM: planetary nebulae: individual: Sh 2-174

\section{Introduction}

Studies of proper motion (PM) require high astrometric accuracy and usually long baselines in time and, preferably, the use of the same equipment in order to keep systematic errors under control. The most recent comprehensive study of the PM of Galactic Planetary Nebulae (PNe) was published by Cudworth (1974) who reported PM for 50 bright nebulae based on positions recorded on photographic plates taken about nine years apart. However, we note that for most of Cudworth's objects no independent confirmation of their PM has been reported so far.

In recent years, thanks both to high astrometric accuracy and extended baselines in time, high quality PM information over the whole sky has become available both from spacebased and ground-based all sky surveys. Resulting catalogues, most notably Hipparcos (Perryman et al. 1997), Tycho-2

Send offprint requests to: $\mathrm{F}$. Kerber, e-mail: florian.kerber@stecf.org
(Høg et al. 1997), USNO-B (Monet et al. 2003) and UCAC 2 (Zacharias et al. 2004) have been published. A new version of the GSC-II, updated with PM information, will also follow very soon (McLean et al., in preparation). All together, these catalogues currently provide the largest and most accurate astrometric database and enable us to embark on a dedicated investigation of PM of Galactic PNe.

The next major improvement in PM research can be expected from the GAIA satellite (Perryman et al. 2001) which will combine superior astrometric accuracy with much fainter limiting magnitudes, bringing many more PNe within its reach. Its results though will only become available more than ten years from now.

Although a straight positional cross-correlation with these catalogues should yield, in principle, the desired PM information for all Galactic PNe, for many of the PNe the compiled positions were not accurate enough for this approach to work. This was one of the drivers of the global reassessment of the 
Table 1. Proper motion data: all values in units of mas $\mathrm{yr}^{-1}$. The ones used for calculating the orbits are given in bold.

\begin{tabular}{l|rr|rr|rr|rr}
\hline \hline PN ID & \multicolumn{2}{|c|}{ Tycho-2 } & \multicolumn{2}{|c|}{ UCAC 2} & & USNO-B & & other source \\
& $\mu_{\alpha} \cdot \cos \delta$ & $\mu_{\delta}$ & $\mu_{\alpha} \cdot \cos \delta$ & $\mu_{\delta}$ & $\mu_{\alpha} \cdot \cos \delta$ & $\mu_{\delta}$ & $\mu_{\alpha} \cdot \cos \delta$ & $\mu_{\delta}$ \\
\hline NGC 7293 & $\ldots$ & $\ldots$ & $\mathbf{3 7 . 3} \pm \mathbf{2 . 5}$ & $\mathbf{- 3 . 9} \pm \mathbf{1 . 8}$ & $38 \pm 8$ & $-8 \pm 6$ & $40.2 \pm 2.8$ & $-1.0 \pm 1.9^{a}$ \\
Sh 2-216 & $25.0 \pm 2.1$ & $-13.0 \pm 2.1$ & $\mathbf{2 1 . 7} \pm \mathbf{1 . 0}$ & $-\mathbf{1 2 . 7} \pm \mathbf{1 . 0}$ & $24 \pm 4$ & $-14 \pm 4$ & $22 \pm 4$ & $-12 \pm 4^{b}$ \\
IC 4593 & $-8.7 \pm \mathbf{1 . 6}$ & $\mathbf{4 . 1} \pm \mathbf{1 . 5}$ & $-6.5 \pm 1.2$ & $2.8 \pm 1.2$ & $-10 \pm 4$ & $4 \pm 4$ & $-7.8 \pm 2.1$ & $-0.8 \pm 3.4^{a}$ \\
Sh 2-174 & $\ldots$ & $\ldots$ & $\ldots$ & $\ldots$ & $-\mathbf{2 8} \pm \mathbf{4}$ & $\mathbf{4} \pm \mathbf{6}$ & $-30.5 \pm 7.1$ & $5.0 \pm 7.1^{c}$ \\
\hline
\end{tabular}

${ }^{a}$ Cudworth (1974), ${ }^{b}$ Cudworth \& Reynolds (1985), ${ }^{c}$ GSC-II Database.

PNe coordinates recently done by Kerber et al. (2003a), which produced a new catalogue of accurate and reliable positions. PM data for a complete sample of PNe will be presented in a subsequent paper (Kerber et al. I, in preparation), which will also contain a detailed comparison with Cudworth's (1974) values. Here we have chosen four PNe, as interesting test cases: namely NGC 7293, Sh 2-216, IC 4593 and Sh 2-174 for which all relevant parameters, including distances, are known with good accuracy. Moreover, since these four objects are representative of different phases of PN evolution, they are perfect test cases to demonstrate how the PM can provide new insight both into the history of a PN and into its interaction with the interstellar medium (ISM). Indeed, Kerber et al. (2002) recently showed how the PM of the central star (CS) of Sh 2-68, the second largest found among PNe, can account for this spectacular example of interaction between the PN and its ambient ISM. Data from the SHASSA H $\alpha$ survey (Gaustad et al. 2001) revealed a tail extending for at least 45 arcmin which is most likely formed by matter stripped off the main nebula in the course of the interaction and which is clearly marking the path of the PN along its Galactic orbit (Kerber et al. 2003b).

\section{Results}

\subsection{Proper motions}

As a reference for our work we have used the Hipparcos, Tycho-2, UCAC 2 and USNO-B catalogues. Comparisons between UCAC 1 and Tycho-2 (Zacharias et al. 2000) and between Tycho-2, Hipparcos and ACT (Astrographic Catalog + Tycho) (Urban et al. 2000) prove the reliability of the PM values across most of the used catalogues. We also note that except for USNO-B, which uses FK5, all the above catalogues are based on the same International Celestial Reference System (ICRS), which makes our PM values directly comparable. Our input PN positions, extracted from the GSC-II (Kerber et al. 2003a), are also tied to this reference frame. This is an important point in the context of systematic errors that could be present in the individual catalogues. Other possible problems are the presence of nebular material close to the CS, or other stars which could limit the accuracy of the positional measurements. For NGC 7293, Sh 2-216 and Sh 2-174 all measurements are of the CS with minimal interference from the nebula. For IC 4593 the PM mesurements refer to the integrated light of the nebula.

Table 1 lists all the independent PM values and their errors for the four PNe considered here, as extracted from the
Tycho-2, UCAC 2 and USNO-B catalogues. Due to their faintness, only two objects, i.e. Sh 2-216 and IC 4593, do appear in the Tycho- 2 catalogue and none is present in the Hipparcos catalogue. Still, for all objects, at least two independent PM measures are available. For Sh 2-174 we included PM values derived by comparing the multi-epoch positions extracted from the GSC-II database, while for the others we have included values from the literature. For USNO-B entries, errors smaller

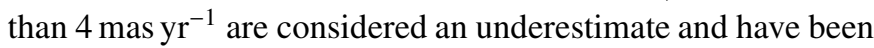
reset to $4 \mathrm{mas} \mathrm{yr}^{-1}$ (Gould 2003). Evidently, the agreement among the PM values is very good in all cases.

\subsection{Orbits and population membership}

When coordinates ( $\alpha$ and $\delta$ ), PM components, distance and radial velocity (Table 2) are all available one can derive the full $3 \mathrm{D}$ information on positions and velocities, and thus the orbits, with respect to the Galactic reference frame. The Galactic velocities $U, V, W$ (Table 2) are also used for analysis of the population membership. For the calculation of the orbits the above parameters are integrated in time using a simplified model of the Galactic gravitational potential. This potential is derived analytically, is symmetrical with respect to the Galactic rotation and is time-independent. The method is described in detail in Pauli et al. (2003). We have to note that our simplified approach does not account for close encounters with other stars which can, of course, drastically change the orbit of the PN at any time. A true orbit simulation would require a full microscopic and time-dependent description of the gravitational environment of the PN, which is of course unrealistic. Thus, our simulated orbits should be considered only representative of the PN's Galactic motion.

The simulated Galactic orbits, the first ever reported for PNe, are shown in Fig. 1. $\rho$ is the distance from the Galactic Center, $z$ is the distance perpendicular to the disk. The plots show the projected Galactic motion over a time span of $2 \mathrm{Gyr}$ with a time step of 1 Myr. They therefore represent a few revolutions of the progenitor star, while the PN phase, which only lasts a tiny fraction of a Galactic orbit, is a point-like event in this representation. Applying the classification criteria of Pauli et al. (2003) yields an unambiguous thin disk membership for NGC 7293 and Sh 2-216, whereas Sh 2-174 belongs to the thick disk. IC 4593 is probably a thick disk object. As detailed in Table 2, all relevant parameters are known with high confidence for all four objects, with the exception of the distance of IC 4593. An analysis of the uncertainties shows that the 
Table 2. Data related to PN orbits.

\begin{tabular}{lcrrrrrrrrr}
\hline \hline PN ID & $\begin{array}{c}\text { Distance } \\
{[\log \mathrm{pc}]}\end{array}$ & $\begin{array}{r}v_{\text {rad }} \\
{\left[\mathrm{km} \mathrm{s}^{-1}\right]}\end{array}$ & $\begin{array}{r}z \\
{[\mathrm{pc}]}\end{array}$ & $\begin{array}{r}\text { Angle } \\
{\left[{ }^{\circ}\right]}\end{array}$ & $\begin{array}{c}U \\
{\left[\mathrm{~km} \mathrm{~s}^{-1}\right]}\end{array}$ & $\begin{array}{c}\sigma(U) \\
{\left[\mathrm{km} \mathrm{s}^{-1}\right]}\end{array}$ & $\begin{array}{c}V \\
{\left[\mathrm{~km} \mathrm{~s}^{-1}\right]}\end{array}$ & $\begin{array}{c}\sigma(V) \\
{\left[\mathrm{km} \mathrm{s}^{-1}\right]}\end{array}$ & $\begin{array}{c}W \\
{\left[\mathrm{~km} \mathrm{~s}^{-1}\right]}\end{array}$ & $\begin{array}{c}\sigma(W) \\
{\left[\mathrm{km} \mathrm{s}^{-1}\right]}\end{array}$ \\
\hline NGC 7293 & $2.33 \pm 0.07^{a}$ & $-27.0 \pm 0.5^{d}$ & -180 & 75 & -34.3 & 5.1 & 225.2 & 1.8 & 10.9 & 3.1 \\
Sh 2-216 & $2.11 \pm 0.03^{a}$ & $11.9 \pm 1.1^{e}$ & 1 & 42 & -4.0 & 1.7 & 236.6 & 3.8 & 18.1 & 4.7 \\
IC 4593 & $3.20 \pm 0.30^{b}$ & $22.0 \pm 0.9^{f}$ & $\approx 1000$ & 88 & -11.1 & 28.5 & 206.1 & 35.2 & 80.0 & 45.4 \\
Sh 2-174 & $2.74 \pm 0.11^{c}$ & $-2.7 \pm 2.0^{g}$ & 175 & 18 & 75.6 & 15.2 & 264.1 & 8.4 & 35.4 & 13.8 \\
\hline
\end{tabular}

${ }^{a}$ Harris et al. (1997), ${ }^{b}$ average from literature, ${ }^{c}$ Napiwotzki $1999,{ }^{d}$ Meaburn et al. (1998), ${ }^{e}$ Tweedy \& Napiwotzki (1992), ${ }^{f}$ Schneider et al. (1983), ${ }^{g}$ Blitz et al. (1982).

classification as thin or thick disk is assured within the stated uncertainties. Also the shape of the orbits do not change appreciably within the errors. A detailed analysis of the error budget and the relative importance of the input parameters will be part of a paper (Kerber et al. II, in preparation) presenting the orbits of our full sample of PNe.

\section{Discussion on individual objects}

\subsection{NGC 7293 (PN G036.1-57.1)}

NGC 7293, the helix nebula, is considered a prototypical PN consisting of a ionized core nebula surrounded by neutral and molecular envelopes (e.g. Speck et al. 2002). The thin disk orbit found in this work confirms that NGC 7293 is a rather normal member of our Galaxy, and that the object can indeed be considered representative of young but fully developed PNe. It is currently located close to its "peri-Galacton" and is moving away from the Galactic plane at an angle of $75^{\circ}$ with respect to Galactic latitude.

\section{2. $S h 2-216$ (PN G158.6+00.7)}

With a trigonometrically measured distance of $130_{-8}^{+9} \mathrm{pc}$ (Harris et al. 1997) Sh 2-216 is the closest and one of the physically largest PNe known. Hence, it is clearly a highly evolved PN. Its central star is located about 0.2 radii off the geometric centre of the huge $(\varnothing \approx 1.6)$ nebula (Tweedy et al. 1995). This fact can only be understood in terms of an interaction with the ISM. This process, described in detail by Borkowski et al. (1990) and Soker et al. (1991), can be summarized as follows: the interaction starts when the density in the shell has dropped - due to expansion - sufficiently to make the ram pressure of the PN shell and the thermal pressure of the ISM comparable. Then, the PN expansion is slowed down upstream and an interaction zone forms. The PN develops an asymmetric shape and instabilities in the shell develop. Later, at even lower densities, the expansion of the PN shell comes to a stop and the CS, which of course is not affected by this interaction, starts to move out off the geometric centre of the PN. First considered a rare phenomenon, ample observational evidence for PN-ISM interaction is now available (Tweedy \& Kwitter 1996; Xilouris et al. 1996; Kerber et al. 2000). In the case of Sh 2-216, PM information (Cudworth \& Reynolds 1985) was already crucial in identifying the CS. A faint red star is located close to the CS, which is marginally resolved on the red
POSS I plate. The agreement of the USNO-B PM with Tycho and UCAC-2, which are based on data with higher spatial resolution, shows that stellar contamination is not a problem here. The available data suggest that the relative velocity between PN and ISM is only a few $\mathrm{km} \mathrm{s}^{-1}$ and at about $5 \mathrm{~cm}^{-3}$ the density is extremely low (Tweedy et al. 1995). Our thin disk orbit of low inclination and eccentricity now confirms that Sh 2-216 is a case of a very mild but highly advanced interaction; from the PM we deduce that the CS started to leave the centre of a quasistatic PN shell about 45000 years ago. For low velocity interaction in the dense ISM very close to the Galactic plane, the PN shell fossilizes and slowly diffuses into the ISM. Since this kind of orbit is typical for vast numbers of stars in the Galactic disk, Sh 2-216 has to be considered proto-typical for the final stage of PN decay and the return of matter to the ISM. Many more such objects are expected to exist in our Galaxy; their low surface brightness makes this final PN phase unobservable for all but very close PNe.

\subsection{IC 4593 (PN G025.3+40.8)}

IC 4593 also shows clear signs of interaction, e.g. a bow shock and fragmented remnants of a halo, downstream (Corradi et al. 2003). In contrast to Sh 2-216, IC 4593 is currently located at about $1 \mathrm{kpc}$ above the Galactic plane and is moving away almost at a $90^{\circ}$ angle. Here the density of the ISM is certainly much lower but a higher relative velocity can easily compensate for this, since it contributes quadratically to the ram pressure. The PM values refer to the integrated light of the PN. As a result of the interaction with the ISM the brightness distribution in the PN body is asymmetric. The agreement between the sources and Cudworth (1974), who measured the CS by virtue of a long-focus refractor and a filter, shows that this asymmetry did not introduce serious problems to the PM determination. IC 4593 is formally classified as a thin disk object but it most likely belongs to the thick disk since its orbits reaches very substantial distances $z$ from the disk. This leads to very substantial relative velocity as the object crosses the Galactic plane at very large angles. Here again, knowledge of the orbit helps to understand the cause for the kind of interaction with the ISM; in this case the high velocity probably causes the massive bow shock of IC 4593. 

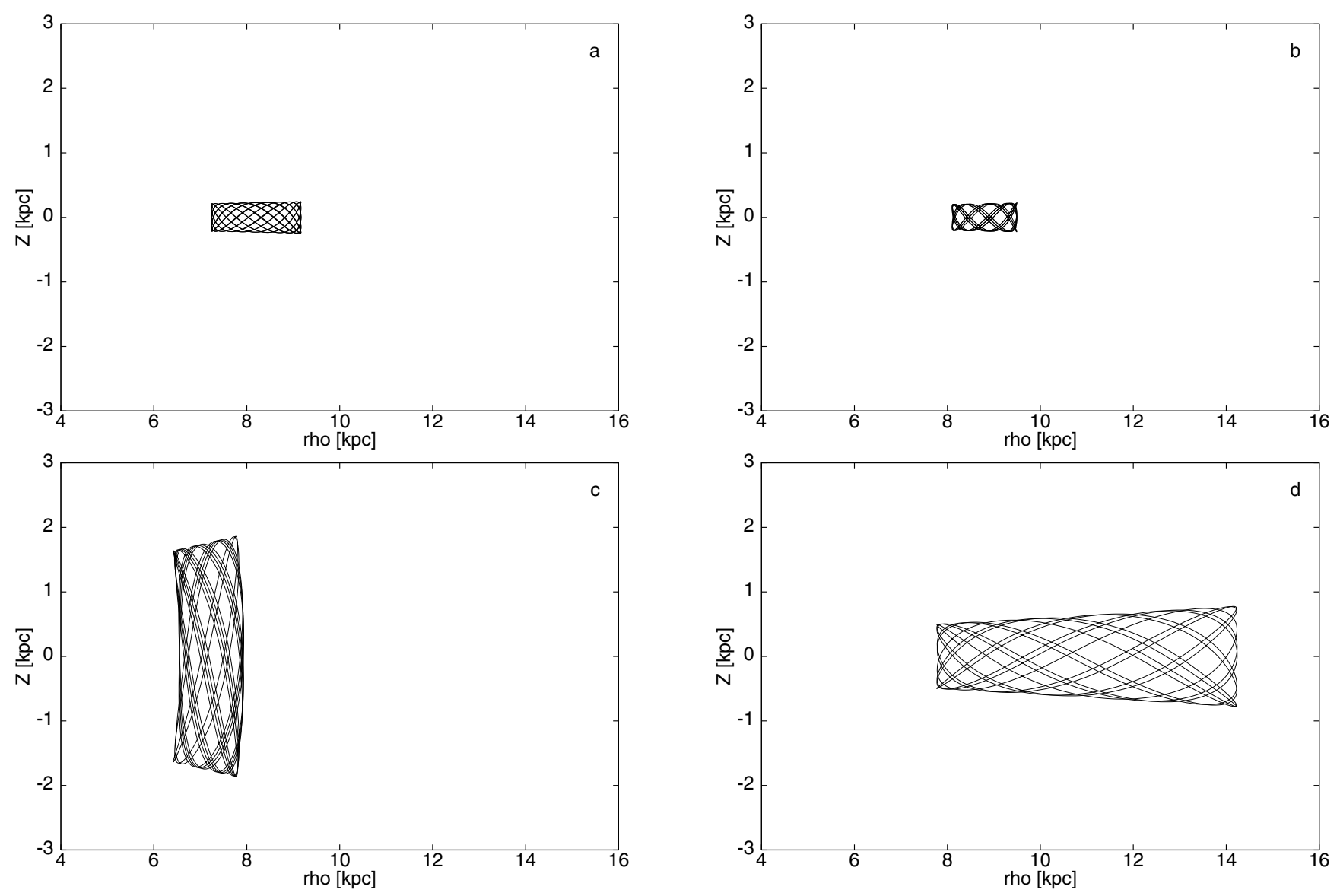

Fig. 1. From upper left to lower right: simulated Galactic orbits for NGC 7293 a) and Sh 2-216 b) both of which have thin disk orbits, IC 4593 c) which is probably thick disk and Sh 2-174 d) which also belongs to the thick disk.

\subsection{Sh 2-174 (PN G120.3+18.3)}

Sh 2-174 has been called "the PN abandoned by its central star" by Tweedy \& Napiwotzki (1994). It is currently the most extreme case of interaction with the ISM, since the CS is actually located outside of the main nebula, see their Fig. 1. With our discovery of the PM (Table 1), we can now conclusively show that the star and the PN are actually linked. By extrapolating back the observed PM, we find that it took the CS about 10000 years to reach a distance of 1.3 radii from the centre. We therefore conclude that the ISM stopped the expansion of the nebula at that time. Our orbit now reveals the reason for this extreme behaviour. It is a thick disk orbit with an unusually high eccentricity. Orbits like this are certainly rare; one might even speculate that at some time in the past it might have been changed by a close encounter with another star, see Sect. 2.2. Since the existence of the thick disk is also attributed to mergers of other stellar systems with the Galaxy, the extreme orbit could also be the result of such a merger. Furthermore such objects spend a fair part of their orbit way outside the solar circle, well protected from discovery. The fact that the PN phase set in not far from the peri-Galacton of course only increases the velocity relative to the ambient ISM, which is moving along a roughly circular orbit. All of the above points combined, make a case like Sh 2-174 a rather unlikely occurrence and it is no surprise that this PN is to date the most extreme case of PN-ISM interaction. In all three cases of interaction with the ISM presented above, the PM we found is in full agreement with predictions by theory concerning the location of the CS within the nebula and the morphology of the PN.

\section{Conclusions}

We have presented PM information for four PNe as early results of a dedicated proper motion survey of Galactic PNe (Kerber et al. I, in preparation). We have then derived the first Galactic orbits of PNe and assigned them membership to the thin or thick disk population. This provides a significant new aspect into the properties of PNe. The analysis of a larger sample of PNe with proper motion (Kerber et al. I and II, in preparation) will enable us to put these findings on a statisticaly solid basis. We have confirmed the motion of the PNe as the root cause for interaction with the ISM in the final stages of PN evolution. Even from this very small sample it can be discerned that eccentricity and inclination of the orbit can have a dramatic influence on the kind of interaction with the ISM. $\mathrm{PM}$ and orbital information will lead to a more quantitiative understanding of the interaction. This opens new insight into a process that governs the return of matter processed in stars back to the ISM, and thereby promotes the chemical evolution of galaxies. 
Acknowledgements. This research has made use of the SIMBAD database and in particular the VizieR Service, operated at CDS, Strasbourg, France. We thank the GSC-II consortium for granting access to data prior to publication. This work was supported by the DFG under grant Na365/2-1(E.M.P.). E.M.P. also wishes to express gratitude to the Studienstiftung des deutschen Volkes for a grant. It is a pleasure to thank the referee, Dr. K. Cudworth for valuable comments that led to a clearer presentation of the case.

\section{References}

Blitz, L., Fich, M., \& Stark, A. A. 1982, ApJS, 49, 183

Borkowski, K. J., Sarazin, C. L., \& Soker, N. 1990, ApJ, 360, 173

Corradi, R. L. M., Schönberner, D., Steffen, M., \& Perinotto, M. 2003, MNRAS, 340, 417

Cudworth, K. 1974, AJ, 79, 1384

Cudworth, K., \& Reynolds, R. J. 1985, PASP, 97, 175

Gaustad, J. E., McCullough, P. R., Rosing, W., \& van Buren, D. 2001, PASP, 113,1326

Gould, A. 2003, AJ, 126, 472

Harris, H. C., Dahn, C. C., Monet, D. G., \& Pier, J. R. 1997, in Planetary Nebulae, ed. H. Habing, \& H. Lamers (Dordrecht: Kluwer) Proc. IAU Symp., 180, 132

Høg, E., Fabricius, C., Makarov, V. V., et al. 2000, A\&A, 355, L27

Kerber, F., Mignani, R. P., Guglielmetti, F., \& Wicenec, A. 2003a, A\&A, 408, 1029

Kerber, F., Guglielmetti, F., Mignani, R. P., \& Roth, M. 2003b, in Planetary Nebulae: Their Evolution and Role in the Universe, ed. S. Kwok, M. Dopita, \& R. Sutherland, Proc. IAU Symp., 209, ASP, San Francisco, 525
Kerber, F., Guglielmetti, F., Mignani, R. P., \& Roth, M. 2002, A\&A, 381, L9

Kerber, F., Furlan, E., Rauch, T., \& Roth, M. 2000, in Asymmetrical Planetary Nebulae II: from Origins to Microstructures, ed. J. H. Kastner, N. Soker, \& S. A. Rappaport, ASP Conf. Ser., 199, 313

Meaburn, J., Clayton, C. A., Bryce, M., et al. 1998, MNRAS, 294, 201

Monet, D. G., Levine, S. E., Canzian, B., et al. 2003, AJ, 125, 984

Napiwotzki, R. 1999, A\&A, 350, 101

Pauli, E.-M., Napiwotzki, R., Altmann, M., et al. 2003, A\&A, 400, 877

Perryman, M. A. C. 2001, Ap\&SS, 280, 1

Perryman, M. A. C., Lindegren, L., Kovalevsky, J., et al. 1997, A\&A, 323, L49

Schneider, S. E., Terzian, Y., Purgathofer, A., \& Perinotto, M. 1983, ApJS, 52, 399

Soker, N., Borkowski, K. J., \& Sarazin, C. L. 1991, AJ, 102, 1381

Speck, A. K., Meixner, M., Fong, D., et al. 2002, AJ, 123, 346

Tweedy, R. W., \& Kwitter, K. B. 1996, ApJS, 107, 255

Tweedy, R. W., Martos, M. A., \& Noriega-Crespo, A. 1995, ApJ, 447, 257

Tweedy, R. W., \& Napiwotzki, R. 1992, MNRAS, 259, 315

Tweedy, R. W., \& Napiwotzki, R. 1994, AJ, 108, 978

Urban, S. E., Wycoff, G. L., \& Makarov, V. V. 2000, AJ, 120, 501

Xilouris, K. M., Papamastorakis, J., Paleologou, E., \& Terzian, Y. 1996, A\&A, 310, 603

Zacharias, N., Urban, S. E., Zacharias, M. I., et al. 2004, AJ, accepted [arXiv: astro-ph/0403060]

Zacharias, N., Zacharias, M. I., Urban, S. E., \& Høg, E. 2000, AJ, 120, 1148 